\title{
Patientenorientierte \\ Versorgungssteuerung im Krankenhaus
}

Ulrich Ronellenfitsch und Matthias Schwarzbach

Inhaltsverzeichnis

4.1 Herausforderungen an die Versorgungssteuerung im Krankenhaus - 70

4.2 Konzept für einen Lösungsansatz - 71

4.3 Klinische Pfade: Definition - 72

4.4 Klinische Pfade: Aufbau, Erstellung und Implementierung - 72

4.5 Evidenz zum Nutzen Klinischer Pfade in der Versorgungssteuerung - 78

4.6 Intersektorale Schnittstellen in Klinischen Pfaden - 79

4.7 Fazit -80

Literatur - 80 


\section{- Zusammenfassung}

In der Patientenversorgung tätige Mitarbeiter erleben häufig einen Konflikt zwischen dem systemimmanent vorgegebenen Ziel einer möglichst wirtschaftlichen Behandlung und der berufsethisch geprägten Bestrebung, ein aus Sicht des Patienten möglichst gutes Behandlungsergebnis zu erreichen. Dieser Konflikt kann durch den Einsatz von Instrumenten zur patientenorientierten Versorgungssteuerung abgemildert werden. Klinische Pfade stellen ein solches Instrument dar. Sie sind interdisziplinäre evidenzbasierte Behandlungspläne für definierte Erkrankungen, Beschwerdebilder oder Prozeduren und geben die idealerweise während der Behandlung durchzuführenden diagnostischen und therapeutischen Maßnahmen vor. Für ihre Erstellung, die Implementierung in den klinischen Alltag und ihren fortwährenden Einsatz ist ein interdisziplinärer und partizipativer Ansatz unabdingbar. Ihr Inhalt ist kontextabhängig anzupassen. Daten zum Nutzen Klinischer Pfade weisen ein eingeschränktes Evidenzlevel auf, da randomisierte Studien methodisch nur schwierig durchführbar sind. Es wäre wünschenswert, wenn Elemente der Intersektoralität in Klinischen Pfaden bislang noch stärker berücksichtigt würden.

Health providers often experience an overt conflict between the system-immanent goal of an economically profitable treatment and the ethically motivated aim of achieving the best outcomes from a patient's perspective. This conflict can be mitigated by employing tools for patient-oriented management of care. Clinical Pathways are an example for such a tool. They can be defined as evidence-based care plans for defined diseases, symptoms, or procedures. They stipulate the diagnostic and therapeutic measures to be ideally performed during the respective treatment. For their design, implementation into clinical routine and continuous usage, a multidisciplinary and participative approach is paramount. Their content should be adapted to the respective con- text. Data regarding their efficacy have a limited evidence level, given that randomized trials are methodologically difficult to conduct. Moreover, Clinical Pathways should account more for intersectoral elements.

\subsection{Herausforderungen an die Versorgungssteuerung im Krankenhaus}

Angesichts der rechtlichen und organisatorischen Vorgaben sowie insbesondere des vom Gesetzgeber beschlossenen Finanzierungsmodells sieht sich die Versorgung stationärer Krankenhauspatienten in Deutschland mit einer Reihe von Herausforderungen konfrontiert. Kliniken sind gezwungen, unter den gegebenen Rahmenbedingungen möglichst wirtschaftlich zu arbeiten. Im Fallpauschalensystem kann dies nur erreicht werden, wenn die Behandlung eines Patienten mit einer definierten Kombination aus Diagnosen und Prozeduren möglichst effizient abläuft. Unnötige und redundante Maßnahmen, die mit Kosten verbunden sind, sollen vermieden werden. Gleichzeitig wird die stationäre Einrichtung systemimmanent motiviert, die Aufenthaltsdauer des Patienten möglichst eng an der im Fallpauschalenkatalog definierten unteren Grenzverweildauer zu orientieren (Thalheimer 2020). Hierdurch sollen die verweildauerabhängigen Kosten gesenkt und die Behandlung möglichst vieler Patienten bei gleichbleibenden Ressourcen gewährleistet werden. Diese rein ökonomische Betrachtungsweise kollidiert nicht selten mit dem Berufsethos von Ärzten und anderen in der unmittelbaren Patientenversorgung tätigen Mitarbeitern (Donabedian 1990; Lenk et al. 2005). Für sie steht im Vordergrund, den Patienten individuell und bestmöglich im Hinblick auf patientenrelevante Outcomes zu behandeln. Diese umfassen verschiedene ,objektive“ Messgrößen wie Mortalität oder Überleben, aber auch „subjektive“ Parameter wie die Lebensqualität, Schmerzfreiheit oder Zufriedenheit mit 


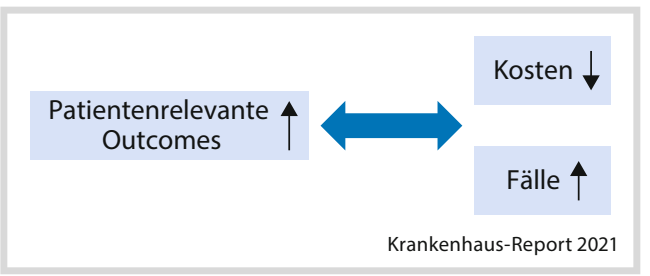

- Abb. 4.1 Spannungsfeld zwischen dem Berufsethos, das die bestmöglichen patientenrelevanten Outcomes erzielen möchte, und vom Gesundheitssystem gemachten pekuniären Vorgaben

der Behandlung. Wirtschaftliche Erwägungen spielen in diesem Kontext allenfalls eine untergeordnete Rolle. Ein Verzicht auf diagnostische oder therapeutische Maßnahmen oder die anzustrebende Verkürzung der Dauer der stationären Krankenhausbehandlung aufgrund pekuniärer Vorgaben ist mit dieser Behandlungsmaxime nicht vereinbar. Dieses Spannungsfeld (•Abb. 4.1) gilt es zur größtmöglichen Zufriedenheit aller Beteiligten, also der direkt in der Patientenversorgung Tätigen, der administrativ im Gesundheitssystem Tätigen sowie der Patienten, aufzulösen.

\subsection{Konzept für einen Lösungsansatz}

Da sich weder die Rahmenbedingungen der stationären Versorgung kurzfristig und grundlegend ändern lassen noch die Maxime der im Sinne patientenrelevanter Outcomes bestmöglichen Behandlung zur Diskussion steht, ist ein integratives Konzept zur Auflösung dieses Spannungsfeldes erforderlich. Dieses Konzept basiert auf der Annahme, dass die Behandlung für eine bestimmte Erkrankung (Beispiel: akute Cholezystitis), ein Beschwerdebild (Beispiel: akuter Oberbauchschmerz) oder eine definierte Prozedur (Beispiel: laparoskopische Cholezystektomie) unter gleichzeitiger Verbesserung der Behandlungsqualität und Outcomes weitgehend standardisiert werden kann (Greenwald et al. 2000). Hierfür ist es unverzichtbar, dass die aktuell verfügbare Evidenz zur jeweiligen Behandlung genauso in die Standardisierung einfließt wie lokal tradierte und bewährte Behandlungsschritte (Beispiel: so genannte „Hausstandards“ und individuell auf den Patienten zugeschnittene Maßnahmen, - Abb. 4.2). Zudem sollte

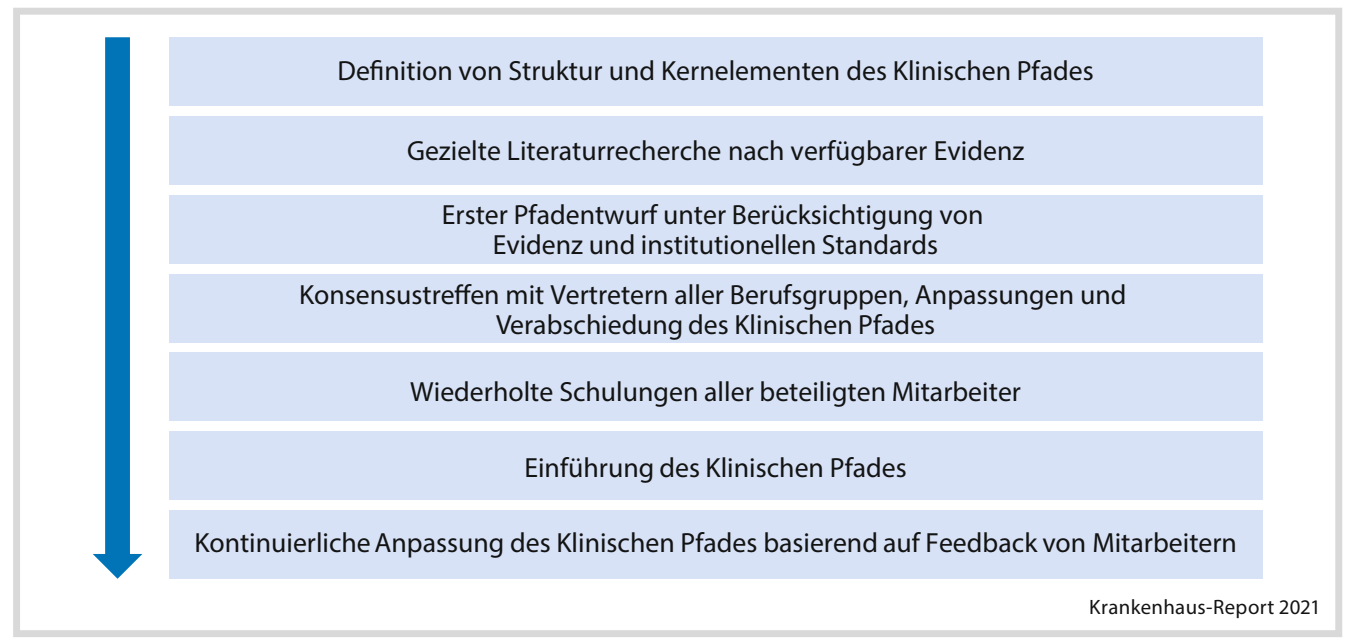

- Abb. 4.2 Empfohlene Sequenz bei der Entwicklung, Implementierung und kontinuierlichem Einsatz eines Klinischen Pfades (adaptiert aus Schwarzbach und Ronellenfitsch 2017) 
das Konzept Schnittstellen zum prästationären ambulanten Sektor sowie zur nachstationären Versorgung im ambulanten oder rehabilitativen Sektor beinhalten. Diese würden im Idealfall die zeitgerechte Planung der stationären Behandlung erlauben, redundante oder kontraindizierte diagnostische und therapeutische Maßnahmen vermeiden und insbesondere auch die poststationäre Weiterbehandlung der Patienten ohne Qualitätsverlust und ohne zeitlichen Verzug gewährleisten. Konkrete Beispiele für durch funktionierende Schnittstellen effektiver durchzuführende Maßnahmen sind die Koordination prästationärer Untersuchungen, die prospektive Planung eines verfügbaren Aufnahme- und ggfs. Operationstermins sowie die Verlegung $\mathrm{zu}$ einer Rehabilitationsmaßnahme ohne eine rein administrativ bedingte zeitliche Latenz (Ronellenfitsch und Schwarzbach 2019). Das beschriebene Konzept vereint Elemente des Case Management mit evidenzbasierter Medizin und Qualitätssicherung. Entscheidend für ein Gelingen des Konzepts und das Erzielen der gewünschten Effekte ist letztendlich seine konkrete Umsetzung in der Behandlung des einzelnen Patienten. Hierfür bietet sich das Behandlungsinstrument der Klinischen Pfade an.

\subsection{Klinische Pfade: Definition}

Klinische Pfade lassen sich zwanglos als zeitlich und inhaltlich strukturierte Ablaufpläne für umschriebene Erkrankungen, Beschwerdebilder oder Prozeduren beschreiben. Ihr Ziel besteht darin, die im Zuge einer Behandlung idealerweise durchzuführenden diagnostischen und therapeutischen Maßnahmen vorzugeben und somit den an der Behandlung beteiligten Personen zu empfehlen. Hierbei soll ein multidisziplinärer und evidenzbasierter Ansatz verfolgt werden (Oberender 2005; Roeder und Küttner 2007).

Gemäß der 2010 publizierten Definition von Kinsman et al. muss ein Klinischer Pfad mindestens die fünf folgenden Kriterien erfüllen (Rotter et al. 2010):

(1) Ist ein multidisziplinärer Behandlungsplan

(2) „Übersetzt“ Leitlinien oder Evidenz in lokale Strukturen

(3) Definiert einzelne Behandlungsschritte in einem Plan oder Algorithmus

(4) Orientiert sich an einer Zeitachse bzw. am Erreichen einzelner Behandlungsschritte

(5) Standardisiert die Behandlung für ein spezifisches klinisches Problem oder einen Eingriff in einer definierten Population

Die Terminologie bezüglich des Behandlungsinstruments ist uneinheitlich. Begriffe wie Klinischer Pfad, Klinikpfad, Behandlungspfad, Patientenpfad oder die aus dem englischen übernommenen Bezeichnungen Clinical Pathway, Critical Pathway, oder Care Pathway werden häufig synonym verwendet (De Bleser et al. 2006). In dieser Arbeit soll einheitlich der Begriff Klinischer Pfad benutzt werden.

\subsection{Klinische Pfade: Aufbau, Erstellung und Implementierung}

Hinsichtlich des Aufbaus Klinischer Pfade gibt es keine einheitliche Vorgabe. Im Jahre 2009 beschrieben Uerlich et al. (2009) mehrere so genannte Entwicklungsstufen Klinischer Pfade:

- Entwicklungsstufe E1: Der Klinische Pfad ergänzt die Patientenakte/-kurve durch zusätzliche Dokumente zur Information des Behandlungsteams, zur Steuerung und Dokumentation der Behandlung sowie ggfs. zur Auswertung der Ergebnisse der Behandlung.

- Entwicklungsstufe E2: Die herkömmlichen Behandlungsdokumente werden im Zuge der Einführung des Klinischen Pfades ganz oder teilweise durch spezielle Dokumente zur Verbesserung von Informationen des Behandlungsteams, Steuerung und Dokumentation der Behandlung und ggfs. zur 
Auswertung der Ergebnisse der Behandlung ersetzt.

- Entwicklungsstufe E3: Der Klinische Pfad ist ein integrierter Bestandteil einer KlinikEDV bzw. des Krankenhausinformationssystems. Informationen sind für das Behandlungsteam situationsgerecht verfügbar. Die Kommunikation und Anordnung diagnostischer und therapeutischer Maßnahmen werden deutlich erleichtert. Die Dokumentation kann automatisch zur Auswertung der Behandlungsergebnisse genutzt werden und erlaubt eine unmittelbare Steuerung während der laufenden klinischen Versorgung.

- Entwicklungsstufe E4: Klinische Pfade sind soweit in die EDV integriert und dergestalt programmiert, dass sie eine umfassende Steuerung und Auswertung variabler Pfaddurchläufe erlauben, mit der Möglichkeit, ein breiteres Patientenspektrum aufzunehmen und Pfadabbrüche zu vermeiden. Aus diesem Ansatz entstand in der Folge auch das Konzept der „Dynamischen Pfade" (Ballies 2017).

Die Definition der einzelnen Entwicklungsstufen wird in der Praxis jedoch nur selten angewandt. In vielen Kliniken werden nach wie vor Klinische Pfade eingesetzt, die formal der Entwicklungsstufe E1 zuzuordnen sind. Der Grund hierfür ist, dass gewohnte, papierbasierte oder elektronische Dokumentationssysteme beibehalten werden sollen und eine Integration Klinischer Pfade in die Krankenhausinformationssysteme oft technisch anspruchsvoll ist und größerer Ressourcen bedarf (Schilling et al. 2006). Bei der Implementierung klinischer Pfade ist letztlich entscheidend, dass die oben genannten fünf Kriterien umgesetzt werden. Die genaue Ausgestaltung des Behandlungsinstruments kann und muss den lokalen infrastrukturellen Gegebenheiten sowie den Vorkenntnissen und der Bereitschaft der an der Behandlung beteiligten Mitarbeiter angepasst werden. Im Zuge der wünschenswerten zunehmenden Digitalisierung der Krankenhäuser sollten jedoch Bestrebungen un- ternommen werden, höhere Entwicklungsstufen der Klinischen Pfade und ihre Integration in Krankenhausinformationssysteme und elektronische Patientenakten $\mathrm{zu}$ erreichen. Dies könnte insgesamt zu einer höheren Akzeptanz und einem vermehrten Einsatz des Instruments Klinischer Pfad führen.

Um Klinische Pfade mit nachhaltigem Erfolg im klinischen Alltag zu etablieren, ist die Art und Weise ihrer Erstellung und Implementierung entscheidend. Vor der Implementierung muss die Auswahl des Patientenkollektivs für einen Klinischen Pfad nach festgelegten Kriterien erfolgen (z. B. Häufigkeit des Krankheitsbildes oder durchschnittliche Behandlungskosten). Die Akzeptanz des Konzepts eines standardisierten klinischen Behandlungsinstruments bei den Mitarbeitern stellt einen entscheidenden Faktor dar. Nur wenn die Mitarbeiter Klinische Pfade annehmen und authentisch vertreten, kommen sie bei der Behandlung des einzelnen Patienten auch tatsächlich zum Einsatz. Sich übergangen fühlende und demotivierte Mitarbeiter werden das Konzept bestenfalls nicht einsetzen und sich ihm schlimmstenfalls aktiv entgegenstellen. Eine von den Autoren an einer chirurgischen Institution durchgeführte qualitative Studie konnte mehrere Erfolgsfaktoren für die Erstellung und Implementierung Klinischer Behandlungspfade identifizieren (De Allegri et al. 2011):

- Interdisziplinarität

- Partizipation der Mitarbeiter am Projekt (,Bottom-up-Ansatz“)

- Zusammengehörigkeitsgefühl innerhalb des Entwicklungsteams, aber auch innerhalb der ganzen Mitarbeiterschaft

- Vorhandensein exponierter Schlüsselpersonen

- Kontinuierliche Schulung der Mitarbeiter

- Kontinuierliches Feedback von allen Mitarbeitern zur stetigen Anpassung der Pfade

Ein zu empfehlender Weg ist es, eine Projektgruppe $\mathrm{zu}$ bilden unter Beteiligung von Repräsentanten aller Disziplinen und Berufsgruppen, die an der Behandlung des jeweiligen 
Krankheits- oder Beschwerdebildes bzw. an der Behandlung vor, während und nach der definierten Prozedur mitwirken. Um den Bottom-up-Ansatz zu realisieren, sollte darauf geachtet werden, dass nicht nur Führungspersonen wie der Klinikdirektor bzw. Chefarzt oder pflegerische Stationsleitungen in der Gruppe repräsentiert sind, sondern auch Vertreter niedrigerer Hierarchiestufen. Für die Erzeugung eines Zusammengehörigkeitsgefühls innerhalb der Gruppe gibt es kein universelles Rezept. Je homogener die Gruppe ist und je inklusiver die Gruppe von ihrem idealerweise im Konsens $\mathrm{zu}$ bestimmenden Leiter geführt wird, desto leichter lässt sich ein solches Gefühl erzeugen. Allerdings muss darauf geachtet werden, dass sich die Gruppe durch zu enge Zusammengehörigkeit nicht gegenüber den „,normalen“ Mitarbeitern abschottet, da für die spätere Implementierung der Klinischen Pfade die Motivation aller Mitarbeiter entscheidend ist. Dem Leiter der Projektgruppe kommt als Schlüsselperson eine besondere Aufgabe zu. Hier sollte eine im gesamten Behandlungsteam breit akzeptierte und respektierte Person ausgewählt werden (Ibarra et al. 1996; Little und Whipple 1996).

Bei der Erstellung Klinischer Pfade muss ein besonderes Augenmerk auf die Translation von aktueller Evidenz und insbesondere von gültigen Leitlinien in lokale Strukturen gelegt werden. Innerhalb der Gruppe sollte ein Konsens bezüglich der Elemente der Behandlung, die im Klinischen Pfad definiert werden, gefunden werden (• Abb. 4.2). Dies lässt sich am Beispiel einer Colonresektion gut illustrieren. Einige mögliche Elemente wären hier: präoperativ notwendige Untersuchungen, perorale Darmvorbereitung, intraoperatives Flüssigkeitsmanagement, intraoperative Platzierung von Drainagen, postoperative Mobilisation, postoperatives Drainagen- und Kathetermanagement, postoperativer Kostaufbau, postoperative Laboruntersuchungen, Entlasskriterien. Diese Elemente sollten dann in einem zweiten Schritt hinsichtlich bestehender Evidenz und gültiger Empfehlungen, aber auch hinsichtlich bestehender institutioneller Stan- dards exploriert werden. Hierzu bietet sich es an, dass eine oder mehrere gut mit dem jeweiligen Behandlungsaspekt vertraute Personen eine systematische Literaturrecherche sowie eine Leitliniensuche durchführen. Die institutionellen Standards sollten von Mitarbeitern mit ausreichend langer Erfahrung im jeweiligen Einsatzgebiet identifiziert werden. Die auf diese Art und Weise ermittelten Behandlungsempfehlungen müssen dann in der Projektgruppe konsentiert werden. Nach Konsensbildung kann dann das Grundgerüst des Klinischen Pfades mit einer Matrix aus einer Zeitachse, auf der nicht zwingend jeder Abschnitt einem gleich großen Zeitraum entsprechen muss, sowie den einzelnen Elementen der Behandlung erstellt und mit detaillierten Inhalten ausgefüllt werden (• Tab. 4.1). Dieser Schritt sollte zur Vermeidung von Redundanzen und Auslassungen von einer definierten Person vorgenommen werden. Die so erstellte Version des Klinischen Pfades muss dann abschließend vor ihrem Inkrafttreten von allen Mitgliedern der Projektgruppe konsentiert werden.

Bevor Klinische Pfade am Patienten eingesetzt werden, müssen die beteiligten Mitarbeiter geschult werden. Diese Schulung sollte idealerweise innerhalb der einzelnen Abteilungen bzw. Bereiche, nach Möglichkeit aber berufsgruppenübergreifend erfolgen. Hierfür bietet es sich an, dass die jeweils in der Projektgruppe vertretenen Personen als Multiplikatoren und „exponierte Schlüsselpersonen“ agieren und die Schulung durchführen. Für das Format der Schulung gibt es keine strikten Vorgaben; es sollte sich an den Bedürfnissen und Erwartungen der Mitarbeiter orientieren. Nach erfolgreicher Schulung aller relevanten Mitarbeitergruppen kann der Klinische Pfad zu einem definierten Stichtag formal eingeführt werden. Für den effektiven dauerhaften Einsatz Klinischer Pfade ist es wichtig, dass diese fortlaufend im Sinne einer Verbesserung angepasst werden (Cheah 2000; Darrikhuma 1999). Hierfür sind zum einen Anregungen aus dem Kreise der Mitarbeiter oder auch von Patienten sehr wichtig. Zum anderen sollte in regelmäßigen Abständen eine Aktualisierung der 


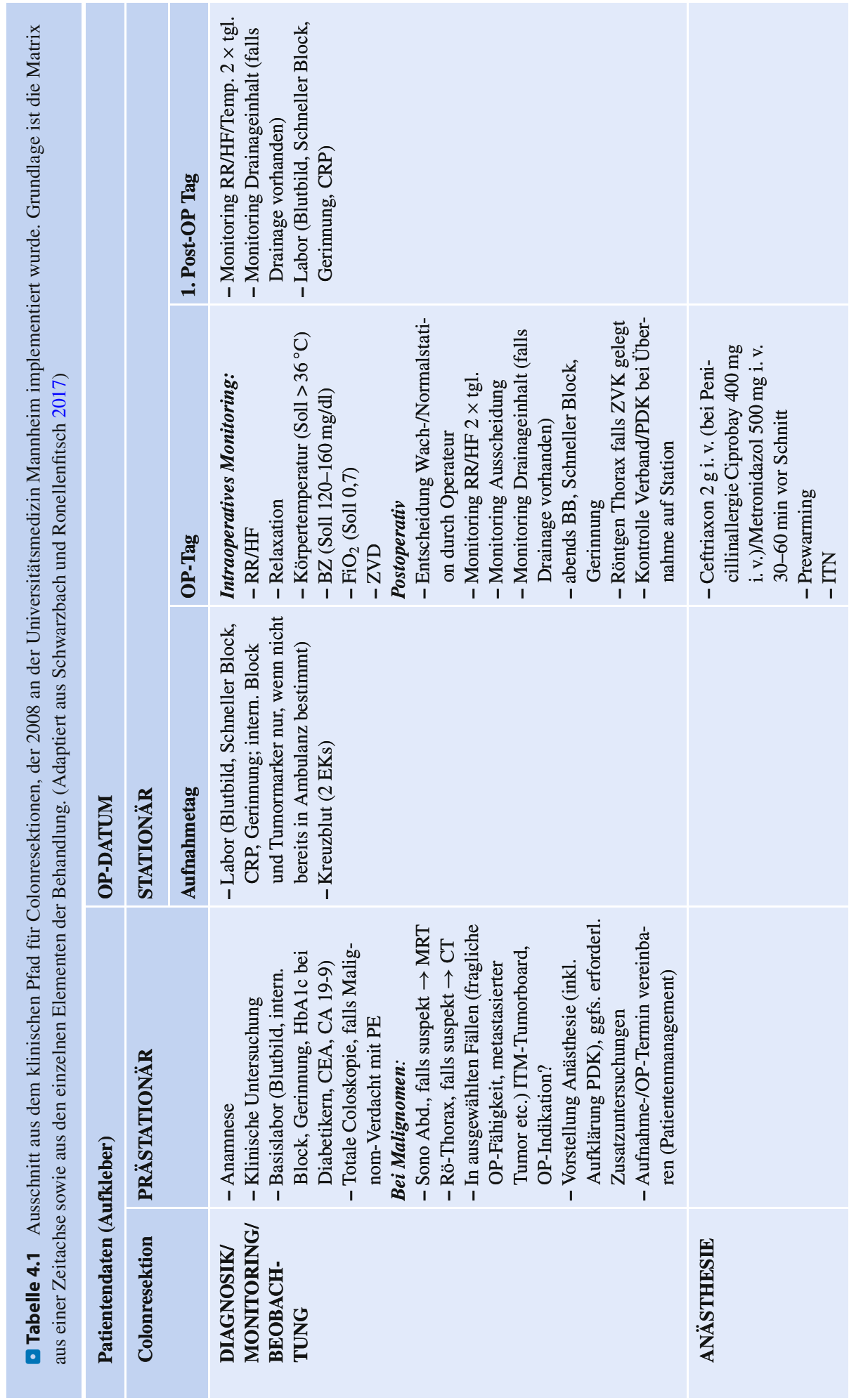




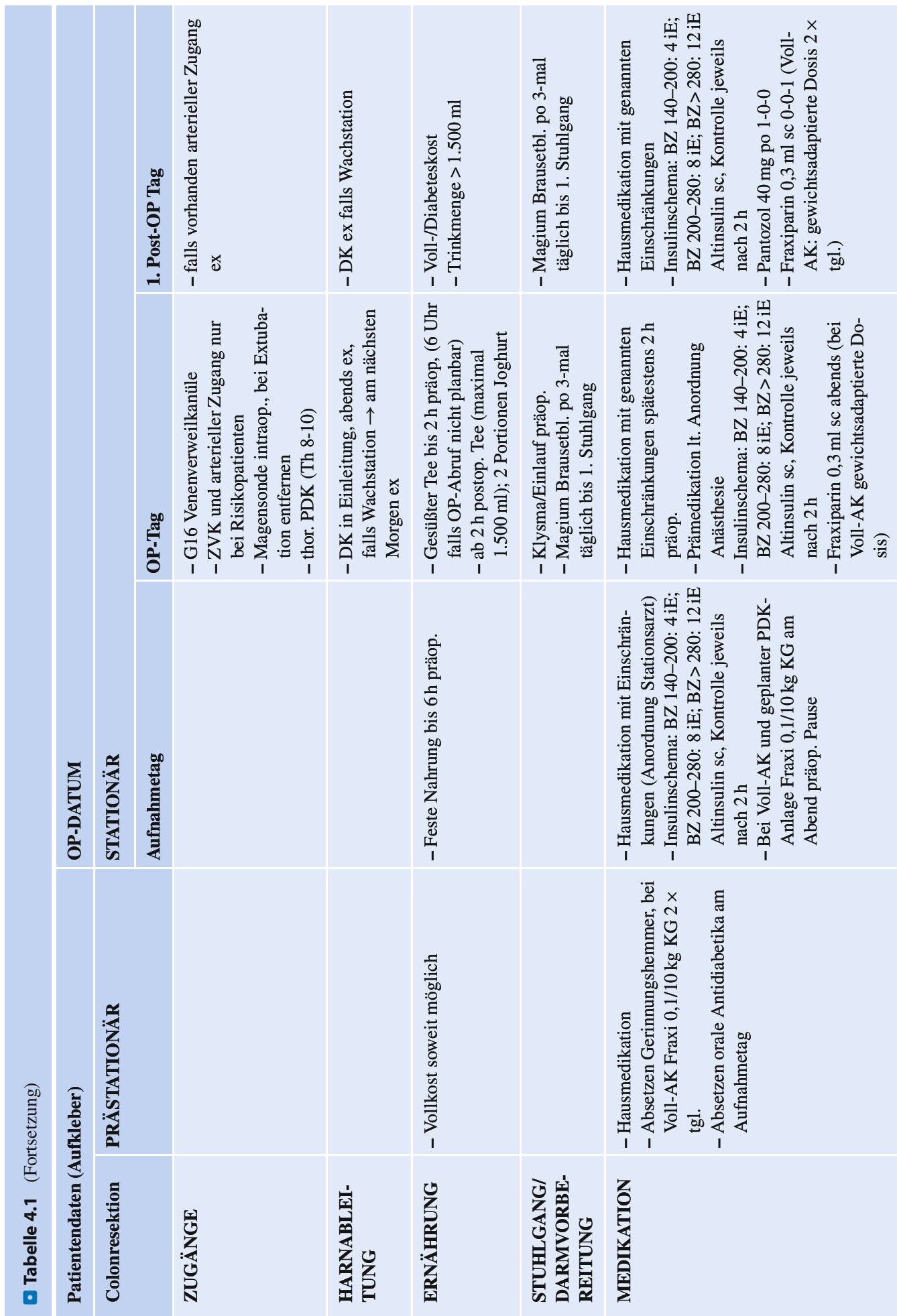




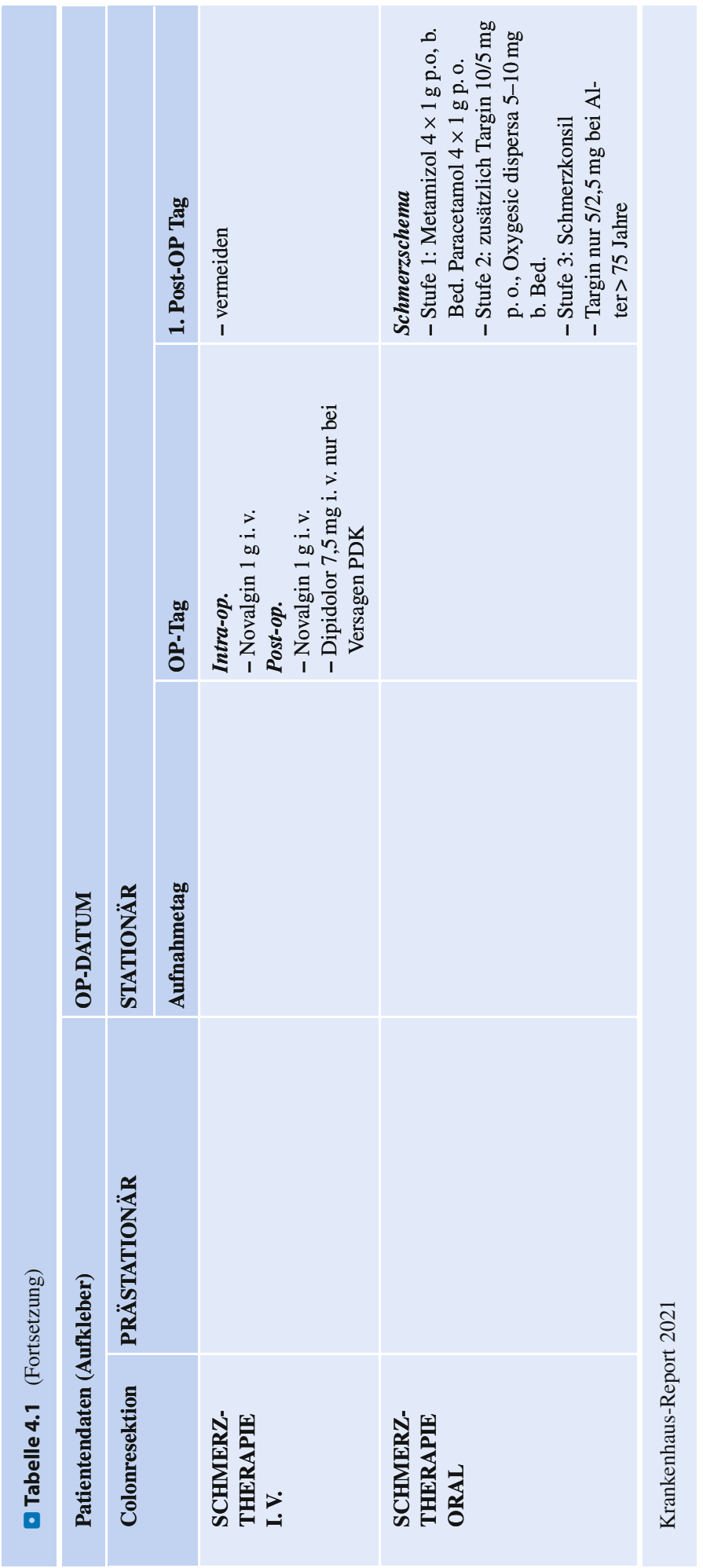


Evidenz- und Leitliniensuche vorgenommen werden. Hierfür bietet es sich an, dass dies die ursprünglich mit der Aufgabe betrauten Personen tun. Es ist aber auch möglich und in bestimmten Situationen sogar wünschenswert, dass diese Aufgabe von anderen Mitarbeitern, die sich beispielsweise besonders bei der täglichen Umsetzung der Klinischen Pfade oder in der Behandlung des jeweiligen Krankheitsbildes engagieren, übernommen wird. Diese Vorgehensweise führt zu einer regelmäßigen Überarbeitung der Klinischen Pfade und zur Verabschiedung neuer Versionen. Dies sollte durch wiederkehrende Schulungsmaßnahmen in den einzelnen Bereichen, wo sich im Zuge der Fluktuation und Rotation auch laufend neue Mitarbeiter einfinden, flankiert werden.

\subsection{Evidenz zum Nutzen Klinischer Pfade in der Versorgungssteuerung}

Im Zuge der Einführung von Fallpauschalen in den USA wurden in den 1980er Jahren Klinische Pfade erstmals eingesetzt. Während sich ihre Anwendung zunächst allerdings weitestgehend auf das Case Management, also eher auf die administrative Versorgungssteuerung beschränkte, hielten sie in der Folge vor allem für operative Behandlungen Einzug in die klinische Routine und unmittelbare Behandlung von Patienten. Nachdem sich ihre Anwendung noch in den 2000er Jahren auf angelsächsische Länder konzentrierte (Vanhaecht et al. 2006), verbreitete sich das Konzept seither in vielen weiteren Gesundheitssystemen. Mit zunehmender Anwendung geriet auch der mögliche Nutzen Klinischer Pfade hinsichtlich relevanter Outcomes der stationären Patientenversorgung in den Focus der Wissenschaft. Eine grundlegende Übersichtsarbeit zeigt, dass Klinische Pfade eine Reihe versorgungsrelevanter Indikatoren insbesondere der Prozess- und Ergebnisqualität beeinflussen (Shabaninejad et al. 2018). Mehrere Übersichtsarbeiten, teils mit Meta-Analy- sen, beleuchteten den Nutzen Klinischer Pfade in spezifischen Behandlungsfeldern. Zusammenfassend ist zu sagen, dass für viele Krankheits- oder Beschwerdebilder und Prozeduren eine Verbesserung der Prozess- und für bestimmte Indikatoren auch der Ergebnisqualität mit dem Einsatz Klinischer Pfade assoziiert ist. Eine 2010 publizierte und innerhalb der Cochrane Collaboration durchgeführte systematische Übersichtsarbeit mit Meta-Analyse schloss nur Studien mit qualitativ höherwertigem Design zu Klinischen Pfaden für ambulante und stationäre Krankenhausbehandlungen ein. Sie zeigte eine geringere Inzidenz von Komplikationen sowie eine verkürzte Verweildauer mit reduzierten Kosten bei gleichbleibender Mortalität und Wiederaufnahmerate für Patienten, die mit Klinischen Pfaden behandelt wurden (Rotter et al. 2010). Eine 2019 publizierte Übersichtsarbeit beschränkte sich auf Klinische Pfade, die durch Gesundheitsinformationstechnologie unterstützt wurden, also solche der Entwicklungsstufe E3 und höher, und zeigte einen positiven Effekt auf patientenrelevante Outcomes, Behandlungsqualität und Ressourcenverbrauch (Neame et al. 2019). Weitere Übersichtsarbeiten untersuchten unter anderem Klinische Pfade für Patienten mit chronisch-obstruktiven Lungenerkrankungen oder Herzinsuffizienz und konnten ebenfalls wünschenswerte Effekte wie eine Reduktion der Inzidenz von Komplikationen oder für das letztgenannte Krankheitsbild auch eine Reduktion der Mortalität feststellen (Kul et al. 2012; Plishka et al. 2019).

Da der Effekt Klinischer Pfade mutmaßlich stark von den Charakteristika des jeweiligen Gesundheitssystems abhängt, ist Evidenz aus Deutschland von besonderem Interesse. Während in der ersten Dekade dieses Jahrtausends sehr viele Einrichtungen Klinische Pfade einführten oder einführen wollten, scheint sich diese Bestrebung in der zweiten Dekade aus einer Vielzahl von Gründen abgeschwächt zu haben. Folglich ist auch die Menge an publizierten wissenschaftlichen Studien zum Einsatz und Nutzen Klinischer Pfade aus Deutschland überschaubar. Ein Behand- 
lungsfeld, in dem jedoch eine Vielzahl von Studien durchgeführt wurde, ist die Chirurgie mit Klinischen Pfaden für chirurgische Krankheitsbilder bzw. spezifische Operationen. Es zeigte sich für viele Eingriffe unter anderem in der Colorektalchirurgie, der bariatrischen Chirurgie, der Thorax- und Transplantationschirurgie sowie für Cholezystektomien und Herniotomien eine signifikante Verbesserung der Prozessqualität und signifikante Kosteneffekte bei weitgehend unveränderter Ergebnisqualität (Hardt et al. 2013; Muehling et al. 2008; Richter-Ehrenstein et al. 2012; Ronellenfitsch et al. 2012; Schwarzbach et al. 2010a, 2010b, 2010c, 2011; Téoule et al. 2019, 2020a, 2020b).

Zusammenfassend besteht derzeit eine hinreichende Evidenz, dass Klinische Pfade die Behandlung von Patienten hinsichtlich unterschiedlicher Teilaspekte zu verbessern vermögen. Ja nach untersuchten Parametern handelt es sich hierbei um objektive oder subjektive direkt patientenrelevante Outcomes wie Morbidität oder Patientenzufriedenheit, aber auch um primär aus der Sicht des Gesundheitssystems wichtige Variablen wie Krankenhausverweildauer oder Kosten. Viele Effekte scheinen jedoch system- und institutionsabhängig zu sein und lassen sich nicht in jeder Studie reproduzieren. Zudem muss konstatiert werden, dass die bislang zum Thema durchgeführten Untersuchungen meist in ihrem Evidenzlevel beschränkt sind, da es sich um nicht-randomisierte Studien handelt. Eine Randomisierung im Kontext der Nutzenbetrachtung Klinischer Pfade gestaltet sich allerdings methodisch schwierig, da bei Randomisierung innerhalb einer Institution ein relevanter „Contamination Bias" zu erwarten wäre (Robinson et al. 2020). Ein gangbarer Weg ist eine ClusterRandomisierung, in der nicht Patienten, sondern komplette Institutionen hinsichtlich des Einsatzes Klinischer Pfade randomisiert werden (Jabbour et al. 2013; Murphy et al. 2006).

\subsection{Intersektorale Schnittstellen in Klinischen Pfaden}

Obwohl sie ein wichtiger Aspekt im Hinblick auf eine effiziente und mit guten Ergebnissen verbundene Behandlung darstellt, kommt der Intersektoralität häufig in Klinischen Pfaden keine ausreichende Bedeutung zu. Idealerweise könnten institutionsspezifische Klinische Pfade von der entsprechenden Einrichtung den Versorgern im niedergelassenen Bereich zur Verfügung gestellt werden. So könnten indizierte und erforderliche Untersuchungen bereits prästationär ambulant erfolgen, während nicht notwendige Maßnahmen möglichst vermieden würden. Auch für die nachstationäre Behandlung und Nachsorge könnten die Empfehlungen der stationären Einrichtung durch den Klinischen Pfad an die ambulanten Versorger übermittelt werden.

Eine Schwierigkeit bezüglich der Intersektoralität besteht darin, dass Kliniken mit einer Vielzahl von ambulanten Versorgern und umgekehrt niedergelassene Einrichtungen mit einer Vielzahl von Kliniken zusammenarbeiten. Dies würde zu mehreren Klinischen Pfaden führen, die eine Praxis je nach Krankenhaus, dem der Patient letztlich zugewiesen wird, einsetzen muss. Bei elektronisch angewendeten Klinischen Pfaden (Entwicklungsstufe E3 und höher) ergibt sich zusätzlich häufig eine EDV-Schnittstellenproblematik. Eine grundsätzliche Frage ist, ob sich ambulante Versorger mit den in Klinischen Pfaden dargelegten Empfehlungen zu Diagnostik und Therapie einverstanden zeigen oder sich hierdurch in ihrer therapeutischen Freiheit eingeschränkt fühlen und daher eine Anwendung möglicherweise ablehnen. Um Synergismen zu nutzen, sollten zukünftig alle Beteiligten auf eine engere intersektorale Verzahnung bei Erstellung, Implementierung und Einsatz Klinischer Pfade hinwirken. 


\subsection{Fazit}

Klinische Pfade stellen ein evidenzbasiertes Instrument zur patientenorientierten Versorgungssteuerung im Krankenhaus dar. Sie können dazu beizutragen, den häufig erlebten Konflikt zwischen geforderter Effizienz und Wirtschaftlichkeit in der Behandlung und dem Berufsethos der Behandelnden, das die subjektiven und objektiven für die Patienten relevanten Ergebnisse der Behandlung und damit das Wohl der Patienten an oberster Stelle sieht, abzumildern. Ihre Auswahl, Erstellung, Implementierung und ihr kontinuierlicher und nachhaltiger Einsatz in der Patientenversorgung erfordern ein strukturiertes und abgestimmtes Vorgehen. Eine Integration Klinischer Pfade in Krankenhausinformationssysteme und elektronische Patientenakten im Zuge der Digitalisierung im Gesundheitswesen könnte dem Einsatz und der Akzeptanz des Behandlungsinstrumentes zugutekommen. Studienergebnisse belegen den kontextabhängigen Nutzen Klinischer Pfade, weisen jedoch ein eingeschränktes Evidenzlevel auf, da randomisierte Studien methodisch nur äußerst schwierig durchführbar sind. Elemente der Intersektoralität sind in vielen Klinischen Pfaden bislang noch nicht ausreichend berücksichtigt.

\section{Literatur}

Ballies A (2017) Dynamische Pfade - Eine Steuerungsmethode akut-stationärer Abläufe als Alternative zum standardisierten Patienten. Cuvillier, Göttingen

Cheah J (2000) Development and implementation of a clinical pathway programme in an acute care general hospital in Singapore. Int J Qual Health Care $12: 403-412$

Darrikhuma IM (1999) Development of a renal transplant clinical pathway: one hospital's journey. AACN Clin Issues 10:270-284

De Allegri M, Schwarzbach M, Loerbroks A, Ronellenfitsch U (2011) Which factors are important for the successful development and implementation of clinical pathways? A qualitative study. BMJ Qual Saf 20:203-208
De Bleser L, Depreitere R, De Waele K, Vanhaecht K, Vlayen J, Sermeus W (2006) Defining pathways. J Nurs Manag 14:553-563

Donabedian A (1990) Quality and cost: choices and responsibilities. J Occup Med 32:1167-1172

Greenwald JA, McMullen HF, Coppa GF, Newman RM (2000) Standardization of surgeon-controlled variables: impact on outcome in patients with acute cholecystitis. Ann Surg 231:339-344

Hardt J, Schwarzbach M, Hasenberg T, Post S, Kienle P, Ronellenfitsch U (2013) The effect of a clinical pathway for enhanced recovery of rectal resections on perioperative quality of care. Int J Colorectal Dis 28:101-126

Ibarra V, Titler MG, Reiter RC (1996) Issues in the development and implementation of clinical pathways. AACN Clin Issues 7:436-447

Jabbour M, Curran J, Scott SD, Guttman A, Rotter T, Ducharme FM, Lougheed MD, McNaughton-Filion ML, Newton A, Shafir M, Paprica A, Klassen T, Taljaard M, Grimshaw J, Johnson DW (2013) Best strategies to implement clinical pathways in an emergency department setting: study protocol for a cluster randomized controlled trial. Implement Sci 8:55

Kul S, Barbieri A, Milan E, Montag I, Vanhaecht K, Panella M (2012) Effects of care pathways on the in-hospital treatment of heart failure: a systematic review. BMC Cardiovasc Disord 12:81

Lenk C, Biller-Andorno N, Alt-Epping B, Anders M, Wiesemann C (2005) Ethics and diagnosis related groups. Dtsch Med Wochenschr 130:1653-1655

Little AB, Whipple TW (1996) Clinical pathway implementation in the acute care hospital setting. J Nurs Care Qual 11:54-61

Muehling BM, Halter GL, Schelzig H, Meierhenrich R, Steffen P, Sunder-Plassmann L, Orend KH (2008) Reduction of postoperative pulmonary complications after lung surgery using a fast track clinical pathway. Eur J Cardiothorac Surg 34:174-180

Murphy AW, Esterman A, Pilotto LS (2006) Cluster randomized controlled trials in primary care: an introduction. Eur J Gen Pract 12:70-73

Neame MT, Chacko J, Surace AE, Sinha IP, Hawcutt DB (2019) A systematic review of the effects of implementing clinical pathways supported by health information technologies. J Am Med Inform Assoc 26:356-363

Oberender P (Hrsg) (2005) Clinical Pathways: Facetten eines neuen Versorgungsmodells. Kohlhammer, Stuttgart

Plishka CT, Rotter T, Penz ED, Hansia MR, Fraser SA, Marciniuk DD (2019) Effects of Clinical Pathways for COPD on Patient, Professional, and Systems Outcomes: A Systematic Review. Chest 156:864-877

Richter-Ehrenstein C, Heymann S, Schneider A, Vargas Hein O (2012) Effects of a clinical pathway 3 years 
after implementation in breast surgery. Arch Gynecol Obstet 285:515-520

Robinson K, Allen F, Darby J, Fox C, Gordon AL, Horne JC, Leighton P, Sims E, Logan PA (2020) Contamination in complex healthcare trials: the falls in care homes (FinCH) study experience. BMC Med Res Methodol 20:46

Roeder N, Küttner T (Hrsg) (2007) Klinische Behandlungspfade. Deutscher Ärzteverlag, Köln

Ronellenfitsch U, Schwarzbach M (2019) Case Management in der Chirurgie. Allg Visceralchir Up2date 13:75-86

Ronellenfitsch U, Schwarzbach M, Kring A, Kienle P, Post S, Hasenberg T (2012) The effect of clinical pathways for bariatric surgery on perioperative quality of care. Obes Surg 22:732-739

Rotter T, Kinsman L, James E, Machotta A, Gothe H, Willis J, Snow P, Kugler J (2010) Clinical pathways: effects on professional practice, patient outcomes, length of stay and hospital costs. Cochrane Database Syst Rev. https://doi.org/10.1002/14651858. CD006632.pub2

Schilling MK, Richter S, Jacob P, Lindemann W (2006) Clinical pathways - first results of a systematic IT-supported application at a surgical department of a university hospital. Dtsch Med Wochenschr 131:962-967

Schwarzbach M, Bönninghoff R, Harrer K, Weiss J, Denz C, Schnülle P, Birck R, Post S, Ronellenfitsch U (2010a) Effects of a clinical pathway on quality of care in kidney transplantation: a non-randomized clinical trial. Langenbecks Arch Surg 395:11-17

Schwarzbach MH, Ronellenfitsch U, Wang Q, Rössner ED, Denz C, Post S, Hohenberger P (2010b) Effects of a clinical pathway for video-assisted thoracoscopic surgery (VATS) on quality and cost of care. Langenbecks Arch Surg 395:333-340

Schwarzbach M, Rössner E, Schattenberg T, Post S, Hohenberger P, Ronellenfitsch U (2010c) Effects of a clinical pathway of pulmonary lobectomy and bilobectomy on quality and cost of care. Langenbecks Arch Surg 395:1139-1146

Schwarzbach M, Hasenberg T, Linke M, Kienle P, Post S, Ronellenfitsch U (2011) Perioperative quality of care is modulated by process management with clini- cal pathways for fast-track surgery of the colon. Int $\mathrm{J}$ Colorectal Dis 26:1567-1575

Schwarzbach M, Ronellenfitsch U (2017) Klinische Behandlungspfade im perioperativen Prozessmanagement. In: Schwenk W (Hrsg) Perioperative Medizin. Thieme, Stuttgart New York, S 517-521

Shabaninejad H, Alidoost S, Delgoshaei B (2018) Identifying and classifying indicators affected by performing clinical pathways in hospitals: a scoping review. Int J Evid Based Healthc 16:3-24

Téoule P, Birgin E, Mertens C, Schwarzbach M, Post S, Rahbari NN, Reißfelder C, Ronellenfitsch U (2020a) Clinical Pathways for Oncological Gastrectomy: Are They a Suitable Instrument for Process Standardization to Improve Process and Outcome Quality for Patients Undergoing Gastrectomy? A Retrospective Cohort Study. Cancers (Basel) 12:434

Téoule P, Kunz B, Schwarzbach M, Birgin E, Rückert F, Wilhelm TJ, Niedergethmann M, Post S, Rahbari NN, Reißfelder C, Ronellenfitsch U (2020b) Influence of Clinical pathways on treatment and outcome quality for patients undergoing pancreatoduodenectomy? A retrospective cohort study. Asian J Surg 43:799-809

Téoule P, Römling L, Schwarzbach M, Birgin E, Rückert F, Wilhelm TJ, Niedergethmann M, Post S, Rahbari NN, Reißfelder C, Ronellenfitsch U (2019) Clinical Pathways For Pancreatic Surgery: Are They A Suitable Instrument For Process Standardization To Improve Process And Outcome Quality Of Patients Undergoing Distal And Total Pancreatectomy? - A Retrospective Cohort Study. Ther Clin Risk Manag 15:1141-1152

Thalheimer M (2020) DRG-Basiswissen - von der Fallpauschale zum Budget. medhochzwei, Heidelberg

Uerlich M, Dahmen A, Tuschy S, Ronellenfitsch U, Eveslage K, Vargas Hein O, Türk-Ihli G, Schwarzbach M (2009) Klinische Pfade - Terminologie und Entwicklungsstufen. Perioper Medizin 1:155-163

Vanhaecht K, Bollmann M, Bower K, Gallagher C, Gardini A, Guezo J, Jansen U, Massoud R, Moody K, Sermeus W, Van Zelm R, Whittle C, Yazbeck A-M, Zander K, Panella M (2006) Prevalence and use of clinical pathways in 23 countries - an international survey by the European Pathway Association. J Integr Care Pathways 10:28-34 
Open Access Dieses Kapitel wird unter der Creative Commons Namensnennung 4.0 International Lizenz (http:// creativecommons.org/licenses/by/4.0/deed.de) veröffentlicht, welche die Nutzung, Vervielfältigung, Bearbeitung, Verbreitung und Wiedergabe in jeglichem Medium und Format erlaubt, sofern Sie den/die ursprünglichen Autor(en) und die Quelle ordnungsgemäß nennen, einen Link zur Creative Commons Lizenz beifügen und angeben, ob Änderungen vorgenommen wurden.

Die in diesem Kapitel enthaltenen Bilder und sonstiges Drittmaterial unterliegen ebenfalls der genannten Creative Commons Lizenz, sofern sich aus der Abbildungslegende nichts anderes ergibt. Sofern das betreffende Material nicht unter der genannten Creative Commons Lizenz steht und die betreffende Handlung nicht nach gesetzlichen Vorschriften erlaubt ist, ist für die oben aufgeführten Weiterverwendungen des Materials die Einwilligung des jeweiligen Rechteinhabers einzuholen.

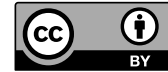

